\title{
On Fuzzy Multiobjective Multi-Item Solid Transportation Problem
}

\author{
Deepika Rani, ${ }^{1}$ T. R. Gulati, ${ }^{1}$ and Amit Kumar ${ }^{2}$ \\ ${ }^{1}$ Department of Mathematics, Indian Institute of Technology Roorkee, Roorkee 247 667, India \\ ${ }^{2}$ School of Mathematics and Computer Applications, Thapar University, Patiala 147 004, India \\ Correspondence should be addressed to Deepika Rani; deepikaiitroorkee@gmail.com
}

Received 9 September 2014; Revised 22 November 2014; Accepted 28 November 2014

Academic Editor: Manuel Lozano

Copyright (c) 2015 Deepika Rani et al. This is an open access article distributed under the Creative Commons Attribution License, which permits unrestricted use, distribution, and reproduction in any medium, provided the original work is properly cited.

\begin{abstract}
A transportation problem involving multiple objectives, multiple products, and three constraints, namely, source, destination, and conveyance, is called the multiobjective multi-item solid transportation problem (MOMISTP). Recently, Kundu et al. (2013) proposed a method to solve an unbalanced MOMISTP. In this paper, we suggest a method, which first converts an unbalanced problem to a balanced one. In one case of an example, while the method proposed by Kundu et al. concludes infeasibility, our method gives a feasible solution.
\end{abstract}

\section{Introduction}

In today's business environment, competition is increasing day by day and each organization aims to find better ways to deliver values to the customers in a cost effective manner within the specified time and fulfill their demands. For this they think of different ideas. One of the ideas can be using different type of transportation modes to save time and money.

Taking into account this factor, the conventional transportation problem proposed by Hitchcock in 1941 [1] was generalized to the transportation problem which takes into account three types of constraints, namely, source, destination, and conveyance constraints instead of only source and destination constraints. This generalized problem is called the solid transportation problem. It was introduced by Schell in 1955 [2]. Solid transportation problem plays a major role in today's business economics as many business situations resemble this. Solution procedure to the solid transportation problem was given by Haley [3].

In many real-world problems, there are situations where several objectives are to be considered and optimized at the same time. Such problems are called multiobjective problems. In multiobjective transportation problems, instead of optimal solution, optimal compromise solution or efficient solution (feasible solution for which no improvement in any objective function is possible without sacrificing at least one of the objective functions) is considered. Zimmermann [4] gave the fuzzy programming technique for the multiobjective transportation problems. B. Liu and Y.-K. Liu [5] presented expected value model for fuzzy programming.

The geometric programming approach for multiobjective transportation problems was considered by Islam and Roy [6]. Gupta et al. [7] proposed a method, called Mehar's method, to find the exact fuzzy optimal solution of unbalanced fully fuzzy multiobjective transportation problems. Gupta and Mehlawat [8] proposed an algorithm for a fuzzy transportation problem to select a new type of coal.

The multiobjective transportation problems in which heterogeneous items are to be transported from different production points to different consumer points using different modes of conveyance are called multiobjective multi-item solid transportation problems (MOMISTPs).

Due to shortage of information, insufficient data, lack of evidence, and so forth, the data for a transportation system such as availabilities, demands, and conveyance capacities are not always exact but can be fuzzy or arbitrary or both. Bector and Chandra [9] have presented a systematic study of fuzzy mathematical programming. The MOMISTPs in which at least one of the parameters is represented by fuzzy 
number [10] are called the fuzzy multiobjective multi-item solid transportation problems.

Genetic algorithm was used by Li et al. [11] to solve multiobjective solid transportation problem (MOSTP) in which only objective function coefficients were taken as fuzzy numbers. Bit et al. [12] applied the fuzzy programming technique to solve MOSTP. Fuzzy MOSTP where all the parameters except decision variables are taken as fuzzy numbers have been solved by Gen et al. [13] and Ojha et al. [14]. Expected-constrained programming for an uncertain solid transportation problem is given by Cui and Sheng [15]. Baidya et al. have discussed the multi-item interval valued solid transportation problem in $[16,17]$. A method to solve multiobjective solid transportation problems in uncertain environment has been presented in [18]. Kundu et al. [19, 20] have proposed methods to solve fuzzy MOMISTP. They have applied the fuzzy programming technique and global criterion method to find the optimal compromise solution.

In this paper a new method is proposed to solve the fuzzy MOMISTP. The application of proposed method is shown by solving two numerical examples in which objective function coefficients, availabilities, and demand parameters are represented by trapezoidal fuzzy numbers. In one case of an example, while the method proposed in [19] concludes no feasible solution, our method gives an optimal compromise solution.

The present paper is organized as follows: Section 2 contains the basic definitions and ranking approach for trapezoidal fuzzy numbers. Mathematical model for the fuzzy MOMISTP is presented in Section 3. The existing method [19] is discussed in Section 4. A new method to solve the fuzzy MOMISTP is proposed in Section 5. To illustrate the proposed method, two numerical examples have been solved in Section 6. Section 7 consists of the results of the two problems with the existing method [19] and that of the proposed method. The interpretation of the results has been given in Section 8. Conclusions are discussed in Section 9.

\section{Preliminaries $[9,21,22]$}

In this section, some basic definitions, arithmetic operations, and ranking approach of trapezoidal fuzzy numbers are presented.

\subsection{Basic Definitions}

Definition 1. A fuzzy number $\widetilde{A}$ defined on the universal set of real numbers $\mathbb{R}$, denoted by $\widetilde{A}=(a, b, c, d)$, is said to be a trapezoidal fuzzy number if its membership function $\mu_{\widetilde{A}}(x)$ is given by

$$
\mu_{\widetilde{A}}(x)= \begin{cases}\frac{(x-a)}{(b-a)}, & a \leq x<b \\ 1, & b \leq x \leq c \\ \frac{(x-d)}{(c-d)}, & c<x \leq d \\ 0, & \text { otherwise. }\end{cases}
$$

Definition 2. A trapezoidal fuzzy number $\widetilde{A}=(a, b, c, d)$ is said to be zero trapezoidal fuzzy number if and only if $a=0$, $b=0, c=0$, and $d=0$.

Definition 3. A trapezoidal fuzzy number $\widetilde{A}=(a, b, c, d)$ is said to be nonnegative trapezoidal fuzzy number if and only if $a \geq 0$.

Definition 4. The support of a fuzzy number $\widetilde{A}$ on $X$ is the crisp set of all $x \in X$ such that $\mu_{\widetilde{A}}(x)>0$.

Definition 5. The core of a fuzzy number $\widetilde{A}$ on $X$ is the crisp set of all $x \in X$ such that $\mu_{\widetilde{A}}(x)=1$.

Remark 6. If, for a trapezoidal fuzzy number $\widetilde{A}=(a, b, c, d)$, $b=c$, then it is called a triangular fuzzy number and is denoted by $(a, b, b, d)$ or $(a, b, d)$ or $(a, c, d)$.

Definition 7. Two trapezoidal fuzzy numbers $\widetilde{A}_{1}=$ $\left(a_{1}, b_{1}, c_{1}, d_{1}\right)$ and $\widetilde{A}_{2}=\left(a_{2}, b_{2}, c_{2}, d_{2}\right)$ are said to be equal if $a_{1}=a_{2}, b_{1}=b_{2}, c_{1}=c_{2}$, and $d_{1}=d_{2}$.

2.2. Arithmetic Operations. Let $\widetilde{A}_{1}=\left(a_{1}, b_{1}, c_{1}, d_{1}\right)$ and $\widetilde{A}_{2}=$ $\left(a_{2}, b_{2}, c_{2}, d_{2}\right)$ be two trapezoidal fuzzy numbers. Then

(i) $\widetilde{A}_{1} \oplus \widetilde{A}_{2}=\left(a_{1}+a_{2}, b_{1}+b_{2}, c_{1}+c_{2}, d_{1}+d_{2}\right)$,

(ii) $\widetilde{A}_{1} \ominus \widetilde{A}_{2}=\left(a_{1}-d_{2}, b_{1}-c_{2}, c_{1}-b_{2}, d_{1}-a_{2}\right)$,

(iii) $k \widetilde{A}_{1}=\left\{\begin{array}{l}\left(k a_{1}, k b_{1}, k c_{1}, k d_{1}\right), k \geq 0 \\ \left(k d_{1}, k c_{1}, k b_{1}, k a_{1}\right), k \leq 0\end{array}\right.$

(iv) $\widetilde{A}_{1} \otimes \widetilde{A}_{2}=(a, b, c, d)$,

where $a=\min \left(a_{1} a_{2}, a_{1} d_{2}, d_{1} a_{2}, d_{1} d_{2}\right), b=\min \left(b_{1} b_{2}, b_{1} c_{2}\right.$, $\left.c_{1} b_{2}, c_{1} c_{2}\right), c=\max \left(b_{1} b_{2}, b_{1} c_{2}, c_{1} b_{2}, c_{1} c_{2}\right)$, and $d=\max \left(a_{1} a_{2}\right.$, $\left.a_{1} d_{2}, d_{1} a_{2}, d_{1} d_{2}\right)$.

2.3. Liou and Wang Ranking Approach for Trapezoidal Fuzzy Numbers. In this paper, we shall use the ranking approach suggested by Liou and Wang [22] to find the crisp value of trapezoidal fuzzy numbers. According to this approach we have the following.

Let $F(\mathbb{R})$ be a set of fuzzy numbers defined on the set of real numbers $\mathbb{R}$ and let $\widetilde{A}=(a, b, c, d) \in F(\mathbb{R})$. Then

$$
\mathfrak{R}(\widetilde{A})=\frac{(a+b+c+d)}{4}
$$

is called a ranking function $\Re: F(\mathbb{R}) \rightarrow \mathbb{R}$, which maps each fuzzy number into the real line.

2.4. Comparison of Trapezoidal Fuzzy Numbers. Let $\widetilde{A}_{1}=$ $\left(a_{1}, b_{1}, c_{1}, d_{1}\right)$ and $\widetilde{A}_{2}=\left(a_{2}, b_{2}, c_{2}, d_{2}\right)$ be two trapezoidal fuzzy numbers. Then

$$
\begin{array}{ll}
\widetilde{A}_{1}>\widetilde{A}_{2} & \text { if } \mathfrak{R}\left(\widetilde{A}_{1}\right)>\mathfrak{R}\left(\widetilde{A}_{2}\right) \\
\widetilde{A}_{1} \prec \widetilde{A}_{2} \quad \text { if } \mathfrak{R}\left(\widetilde{A}_{1}\right)<\mathfrak{R}\left(\widetilde{A}_{2}\right) \\
\widetilde{A}_{1} \approx \widetilde{A}_{2} \quad \text { if } \mathfrak{R}\left(\widetilde{A}_{1}\right)=\mathfrak{R}\left(\widetilde{A}_{2}\right) .
\end{array}
$$




\section{Mathematical Model of Multiobjective Multi-Item Solid Transportation Problem [19]}

A multiobjective multi-item solid transportation problem with $R$ objectives, in which $l$ different items are to be transported from $m$ sources $\left(S_{i}\right)$ to $n$ destinations $\left(D_{j}\right)$ via $K$ different conveyances, can be formulated as follows:

$$
\begin{array}{ll}
\text { Minimize } & \left(Z_{1}, Z_{2}, \ldots, Z_{R}\right) \\
\text { subject to } & \sum_{j=1}^{n} \sum_{k=1}^{K} x_{i j k}^{p} \leq \tilde{a}_{i}^{p} ; \quad 1 \leq i \leq m, \quad 1 \leq p \leq l \\
& \sum_{i=1}^{m} \sum_{k=1}^{K} x_{i j k}^{p} \geq \widetilde{b}_{j}^{p} ; \quad 1 \leq j \leq n, \quad 1 \leq p \leq l \\
& \sum_{p=1}^{l} \sum_{i=1}^{m} \sum_{j=1}^{n} x_{i j k}^{p} \leq \tilde{e}_{k} ; \quad 1 \leq k \leq K \\
x_{i j k}^{p} \geq 0, \quad \forall i, j, k, p,
\end{array}
$$

where $\tilde{a}_{i}^{p}$ is the fuzzy availability of item $p$ at $S_{i}, \widetilde{b}_{j}^{p}$ is the fuzzy demand of item $p$ at $D_{j}, \widetilde{c}_{i j k}^{t p}$ is the fuzzy penalty for transporting one unit of item $p$ from $S_{i}$ to $D_{j}$ via $k$ th conveyance for $t$ th objective $Z_{t}, \widetilde{e}_{k}$ is the total fuzzy capacity of $k$ th conveyance, $x_{i j k}^{p}$ is the quantity of item $p$ to be transported from $S_{i}$ to $D_{j}$ using $k$ th conveyance, and $Z_{t}=$ $\sum_{p=1}^{l} \sum_{i=1}^{m} \sum_{j=1}^{n} \sum_{k=1}^{K}\left(\widetilde{c}_{i j k}^{t p} x_{i j k}^{p}\right), 1 \leq t \leq R$.

For the above problem to be balanced, it should satisfy the following conditions.

(i) Total availability of an item at all sources should be equal to its demand at all the destinations.

(ii) Overall availability of all the items at all the sources, overall demand of all the items at all the destinations, and total conveyance capacity should be equal.

Mathematically, it means

(i) $\mathfrak{R}\left(\sum_{i=1}^{m} \tilde{a}_{i}^{p}\right)=\mathfrak{R}\left(\sum_{j=1}^{n} \tilde{b}_{j}^{p}\right), 1 \leq p \leq l$,

(ii) $\mathfrak{R}\left(\sum_{p=1}^{l} \sum_{i=1}^{m} \tilde{a}_{i}^{p}\right)=\mathfrak{R}\left(\sum_{p=1}^{l} \sum_{j=1}^{n} \widetilde{b}_{j}^{p}\right)=\mathfrak{R}\left(\sum_{k=1}^{K} \widetilde{e}_{k}\right)$.

\section{The Existing Method}

Recently, Kundu et al. [19] proposed a method to solve the fuzzy MOMISTPs. Their method does not require to convert the unbalanced MOMISTP to a balanced one. The first step is to defuzzify the fuzzy parameters (availability, demand, conveyance capacity, and objective function coefficients). On defuzzification, the problem gets converted to the crisp multiobjective multi-item transportation problem. For defuzzification, the authors have used two different methods. They observed out that the expected value model does not give a feasible solution. It may be due to the fact that the expected value method gives $\mathfrak{R}$ (total availability) $=122.25$,
$\mathfrak{R}$ (total demand) $=116$, and $\mathfrak{R}$ (total conveyance capacity) $=104.5$. Thus $\mathfrak{R}$ (total conveyance capacity) $<\mathfrak{R}$ (total demand), which implies infeasibility.

In this paper, we propose a method for MOMISTPs. The method first converts an unbalanced problem to a balanced one. Therefore the expected value model gives a feasible solution. This is then used to obtain its optimal compromise solution. Our method gives better value of the objective function than that obtained in [19].

\section{The Proposed Method}

In this section a new method has been proposed to find the optimal compromise solution of fuzzy MOMISTP in which all the parameters except the decision variables are represented by trapezoidal fuzzy numbers. The method consists of the following steps.

Step 1. Check whether the problem under consideration is balanced or not (according to the definition given in Section 3).

For this, find $\sum_{i=1}^{m} \widetilde{a}_{i}^{p}$ and $\sum_{j=1}^{n} \widetilde{b}_{j}^{p}$ for each $p, 1 \leq p \leq l$. Check the equality of the two as given in Definition 7 .

Case 1. If $\Re\left(\sum_{i=1}^{m} \tilde{a}_{i}^{p}\right)=\mathfrak{R}\left(\sum_{j=1}^{n} \tilde{b}_{j}^{p}\right), 1 \leq p \leq l$, that is, the problem is balanced, then go to Step 2 .

Case 2. If $\mathfrak{R}\left(\sum_{i=1}^{m} \tilde{a}_{i}^{p}\right) \neq \Re\left(\sum_{j=1}^{n} \widetilde{b}_{j}^{p}\right)$ for any $p, 1 \leq p \leq l$, then, to make the problem balanced, proceed according to the following subcases. One or both of these subcases may apply.

Case 2.1. Check if $\mathfrak{R}\left(\sum_{i=1}^{m} \tilde{a}_{i}^{p}\right)<\mathfrak{R}\left(\sum_{j=1}^{n} \widetilde{b}_{j}^{p}\right)$. Say this happens for one or more items. Then introduce a dummy source with fuzzy availabilities of these items equal to any nonnegative trapezoidal fuzzy number whose ranks are equal to the difference of ranks of total demand and total availability of the corresponding items. Assume the unit fuzzy cost of transporting these items from this dummy source to any of the destinations via any of the conveyances as zero trapezoidal fuzzy number.

Case 2.2. Check if $\mathfrak{R}\left(\sum_{i=1}^{m} \tilde{a}_{i}^{p}\right)>\mathfrak{R}\left(\sum_{j=1}^{n} \widetilde{b}_{j}^{p}\right)$ for one or more items. Then introduce a dummy destination similar to dummy source in Case 2.1.

Now proceed to Step 2.

Step 2. Using Step 1 , we have $\mathfrak{R}\left(\sum_{p=1}^{l} \sum_{i=1}^{u} \tilde{a}_{i}^{p}\right)=$ $\mathfrak{R}\left(\sum_{p=1}^{l} \sum_{j=1}^{v} \tilde{b}_{j}^{p}\right)=V$ (say) (where $u=m$ or $m+1$ and $v=n$ or $n+1)$.

Case 1. Consider $V=\mathfrak{R}\left(\sum_{k=1}^{K} \widetilde{e}_{k}\right)$; that is, overall availability, overall demand, and overall conveyance capacity are equal; then go to Step 3.

Case $2\left(V-\mathfrak{R}\left(\sum_{k=1}^{K} \widetilde{e}_{k}\right)=\alpha \neq 0\right)$. Then one of the following conditions will hold.

Case $2.1(\alpha>0)$. Then introduce a dummy conveyance having fuzzy capacity equal to any nonnegative fuzzy number 
whose rank is equal to $\alpha$. Assume the unit fuzzy cost of transportation from all the source to all the destination via this added dummy conveyance as zero trapezoidal fuzzy number.

Case $2.2(\beta=-\alpha>0)$. Then check whether in Step 1 a dummy source and/or a dummy destination were introduced or not.

Case 2.2.1. If both a dummy source and a dummy destination were introduced, then increase the overall fuzzy availability and fuzzy demand of the already added dummy source and dummy destination by a nonnegative fuzzy number whose rank is $\beta$.

Case 2.2.2. If only a dummy source is introduced in Step 1, then increase its overall availability by a nonnegative fuzzy number having rank $\beta$ and also add a dummy destination having this increased demand.

Case 2.2.3. If only a dummy destination is introduced in Step 1 , then increase its overall demand by a nonnegative fuzzy number having rank $\beta$ and also add a dummy source having this increased availability.

Case 2.2.4. If neither a dummy source nor a dummy destination was introduced in Step 1, then add a dummy source as well as a dummy destination having availability and demand equal to any nonnegative fuzzy number whose rank is $\beta$.

Assume the unit fuzzy cost of transportation from the newly introduced source to all the destinations via any of the conveyance as zero trapezoidal fuzzy number.

The problem is balanced now.

Step 3. The next step is to convert the balanced fuzzy MOMISTP problem, obtained by using Steps 1 and 2, into a crisp MOMISTP. We will discuss two different methods for this.

Step 3.1 (using rank of the fuzzy number). Formulate the crisp model by obtaining the rank of the objective function coefficients as well as the constraints, as shown in the following:

$$
\begin{array}{ll}
\text { Minimize } & \left(Z_{1}, Z_{2}, \ldots, Z_{R}\right) \\
\text { subject to } & \sum_{j=1}^{n} \sum_{k=1}^{K} x_{i j k}^{p}=\Re\left(\widetilde{a}_{i}^{p}\right), \quad 1 \leq i \leq m, \quad 1 \leq p \leq l \\
& \sum_{i=1}^{m} \sum_{k=1}^{K} x_{i j k}^{p}=\Re\left(\widetilde{b}_{j}^{p}\right), \quad 1 \leq j \leq n, \quad 1 \leq p \leq l \\
& \sum_{p=1}^{l} \sum_{i=1}^{m} \sum_{j=1}^{n} \sum_{k=1}^{K} x_{i j k}^{p}=\Re\left(\widetilde{e}_{k}\right), \quad 1 \leq k \leq K \\
x_{i j k}^{p} \geq 0, \quad \forall i, j, k, p,
\end{array}
$$

where

$$
Z_{t}=\sum_{p=1}^{l} \sum_{i=1}^{m} \sum_{j=1}^{n} \sum_{k=1}^{K} \mathfrak{R}\left(\widetilde{c}_{i j k}^{t p}\right) x_{i j k}^{p}, \quad 1 \leq t \leq R .
$$

This method provides a crisp optimal compromise solution.

Step 3.2 (using the concept of minimum of fuzzy number)

Step 3.2.1 (brief overview of the method). Since a fuzzy number $\widetilde{Z}$ cannot be minimized directly, Buckley et al. [23] proposed to find $\min \widetilde{Z}$ by first converting it to a multiobjective problem:

$$
\min \widetilde{Z}=\left(\max A_{L}, \min C, \min A_{U}\right),
$$

where $A_{L}, A_{U}$ are the areas under the graph of membership function of $\widetilde{Z}$ to the left and right of the center of core $C$.

In the above multiobjective problem, we wish to

(i) maximize the possibility of getting values less than $C$,

(ii) minimize the possibility of getting values more than C.

This multiobjective problem is then changed to the following single objective optimization problem:

$$
\min \left\{\lambda_{1}\left[M-A_{L}\right]+\lambda_{2} C+\lambda_{3} A_{U}\right\}
$$

where $M>0$ is so large such that $\max A_{L}$ is equivalent to $\min \left[M-A_{L}\right] ; \lambda_{i}>0, i=1,2,3$, and $\lambda_{1}+\lambda_{2}+\lambda_{3}=1$. The values of $\lambda_{i}$ 's depend upon the decision maker's choice.

Step 3.2.2 (to calculate the values of $A_{L}, C$, and $A_{R}$ ). Since we have taken the objective function coefficients to be trapezoidal fuzzy numbers, let

$$
\widetilde{c}_{i j k}^{t p}=\left(c_{i j k}^{1 t p}, c_{i j k}^{2 t p}, c_{i j k}^{3 t p}, c_{i j k}^{4 t p}\right)
$$

Therefore, each objective function is also a trapezoidal fuzzy number

$$
\widetilde{Z_{t}}=\left(Z_{t}^{1}, Z_{t}^{2}, Z_{t}^{3}, Z_{t}^{4}\right)
$$

where $Z_{t}^{r}=\sum_{p=1}^{l} \sum_{i=1}^{m} \sum_{j=1}^{n} \sum_{k=1}^{K}\left(c_{i j k}^{r t p} x_{i j k}^{p}\right), 1 \leq r \leq 4$. The values of $A_{L}, C$, and $A_{R}$ can be calculated as follows:

$$
C=\frac{Z_{t}^{2}+Z_{t}^{3}}{2}, \quad A_{L}=\frac{Z_{t}^{3}-Z_{t}^{1}}{2}, \quad A_{R}=\frac{Z_{t}^{4}-Z_{t}^{2}}{2}
$$




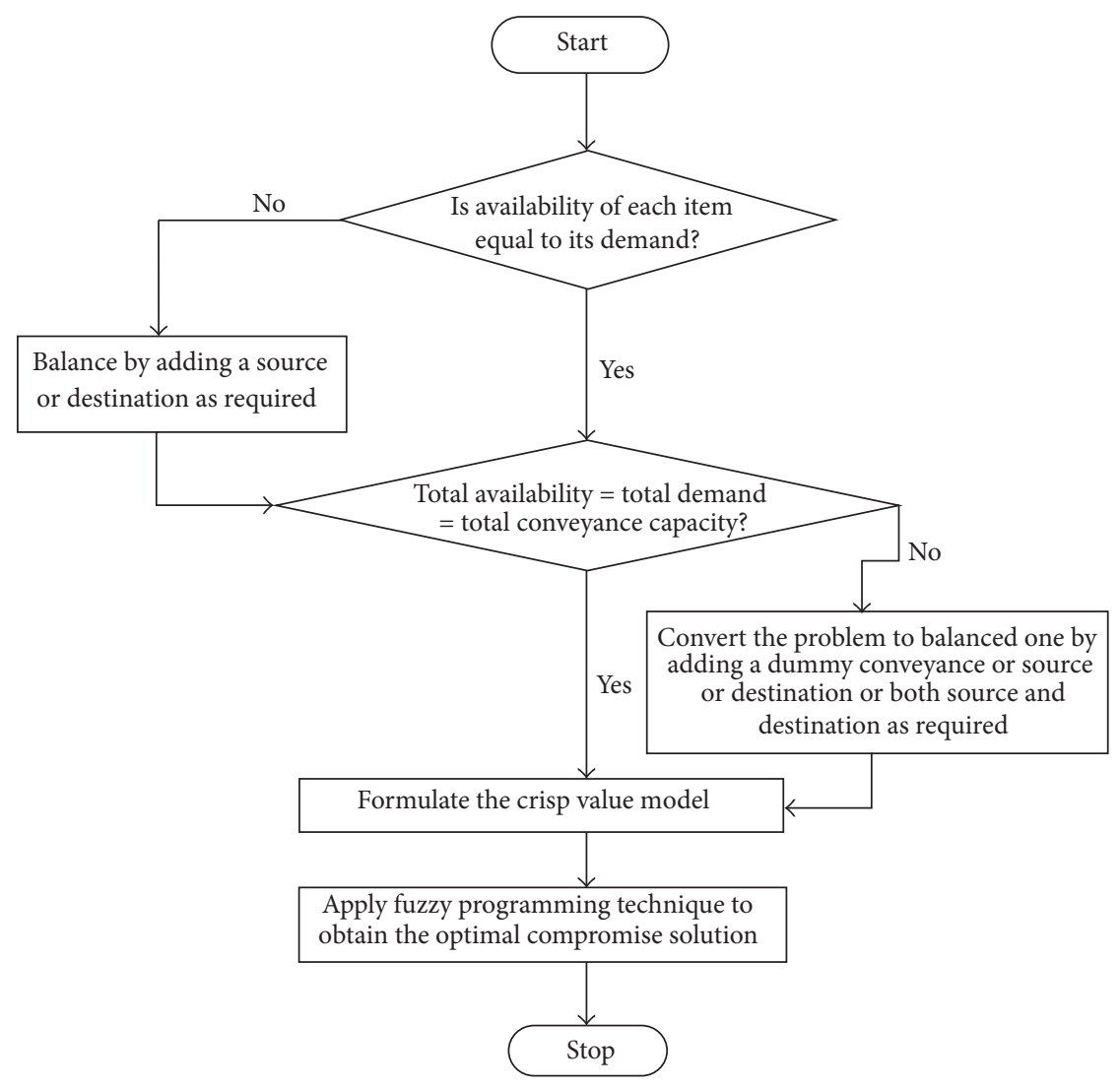

FIGURE 1: Flow chart for the proposed method.

Now, formulate the crisp model as shown in the following:

$$
\begin{array}{ll}
\text { Minimize } & \left(Z_{1}, Z_{2}, \ldots, Z_{R}\right) \\
\text { subject to } & \sum_{j=1}^{n} \sum_{k=1}^{K} x_{i j k}^{p}=\Re\left(\widetilde{a}_{i}^{p}\right), \quad 1 \leq i \leq m, \quad 1 \leq p \leq l \\
& \sum_{i=1}^{m} \sum_{k=1}^{K} x_{i j k}^{p}=\Re\left(\widetilde{b}_{j}^{p}\right), \quad 1 \leq j \leq n, \quad 1 \leq p \leq l \\
& \sum_{p=1}^{l} \sum_{i=1}^{m} \sum_{j=1}^{n} \sum_{k=1}^{K} x_{i j k}^{p}=\Re\left(\widetilde{e}_{k}\right), \quad 1 \leq k \leq K \\
x_{i j k}^{p} \geq 0, \quad \forall i, j, k, p,
\end{array}
$$

where

$$
\begin{array}{r}
Z_{t}=\left\{\begin{array}{r}
\left.\lambda_{1}\left[M-A_{L}\left(Z_{t}\right)\right]+\lambda_{2} C\left(Z_{t}\right)+\lambda_{3} A_{U}\left(Z_{t}\right)\right\}, \\
1 \leq t \leq R
\end{array}\right. \\
\lambda_{i}>0, \quad i=1,2,3, \lambda_{1}+\lambda_{2}+\lambda_{3}=1 .
\end{array}
$$

Step 4. Solve the crisp multiobjective linear programming problem, obtained in Step 3, by any of the existing multiobjective programming techniques and find the optimal compromise solution.

Remark 8. There are several defuzzification methods (e.g., $[22,24])$ to defuzzify the fuzzy numbers. But in this paper, we will use the defuzzification method given by Liou and Wang [22] as explained in Section 2.4, called the rank value. One can use any of the defuzzification methods.

A flowchart of the proposed method is shown in Figure 1.

\section{Numerical Example}

6.1. Example 1. Consider the fuzzy MOMISTP solved by Kundu et al. [19] in which two items are to be transported via two different conveyances from two sources to three destinations and consisting of two different objective functions. Solve the problems to find the amount of goods to be shipped from source(s) to destination(s) so that the total demand at all the destinations is met at the minimum total cost and in minimum time possible. Data of the problem is as shown in Tables 1, 2, 3, 4, and 5.

From Table 5, we find that, for the first item, total availability $\left(\sum_{i=1}^{2} \tilde{a}_{i}^{1}\right)=(21,24,26,28) \oplus(28,32,35,37)=$ $(49,56,61,65)$ and total demand $\left(\sum_{j=1}^{3} \widetilde{b}_{j}^{1}\right)=(14,16,19,22) \oplus$ $(17,20,22,25) \oplus(12,15,18,21)=(43,51,59,68)$.

Similarly, for the second item, total availability $\left(\sum_{i=1}^{2} \tilde{a}_{i}^{2}\right)=$ $(57,62,67,72)$ and total demand $\left(\sum_{j=1}^{3} \widetilde{b}_{j}^{2}\right)=(51,58,63,71)$. After calculating the rank for all these, we find that

$$
\mathfrak{R}\left(\sum_{i=1}^{2} \widetilde{a}_{i}^{l}\right)>\mathfrak{R}\left(\sum_{j=1}^{3} \widetilde{b}_{j}^{l}\right), \quad l=1,2 .
$$


TABLE 1: Unit penalties of transportation for item 1 in the first objective.

(a)

\begin{tabular}{lccc}
\hline \multicolumn{4}{c}{ Conveyance $k=1$} \\
Sources & $D_{1}$ & Destinations \\
& $(5,8,9,11)$ & $(4,6,9,11)$ & $D_{3}$ \\
\hline$S_{1}$ & $(8,10,13,15)$ & $(6,7,8,9)$ & $(11,13,15,14,16)$ \\
$S_{2}$ &
\end{tabular}

(b)

\section{Conveyance $k=2$}

\begin{tabular}{lccc} 
Sources & \multicolumn{3}{c}{ Destinations } \\
& $D_{1}$ & $D_{2}$ & $D_{3}$ \\
\hline$S_{1}$ & $(9,11,13,15)$ & $(6,8,10,12)$ & $(7,9,12,14)$ \\
$S_{2}$ & $(10,11,13,15)$ & $(6,8,10,12)$ & $(14,16,18,20)$ \\
\hline
\end{tabular}

TABLE 2: Unit penalties of transportation for item 2 in the first objective.

(a)

\section{Conveyance $k=1$}

\begin{tabular}{lccc} 
Sources & \multicolumn{3}{c}{ Destinations } \\
& $D_{1}$ & $D_{2}$ & $D_{3}$ \\
\hline$S_{1}$ & $(9,10,12,13)$ & $(5,8,10,12)$ & $(10,11,12,13)$ \\
$S_{2}$ & $(11,13,14,16)$ & $(7,9,12,14)$ & $(12,14,16,18)$ \\
\hline
\end{tabular}

(b)

Conveyance $k=2$

\begin{tabular}{lccc} 
Sources & \multicolumn{3}{c}{ Destinations } \\
& $D_{1}$ & $D_{2}$ & $D_{3}$ \\
\hline$S_{1}$ & $(11,13,14,15)$ & $(6,7,9,11)$ & $(8,10,11,13)$ \\
$S_{2}$ & $(14,16,18,20)$ & $(9,11,13,14)$ & $(13,14,15,16)$ \\
\hline
\end{tabular}

So to balance the problem add a dummy destination $D_{4}$ with demands of items 1 and 2 equal to any fuzzy number whose ranks are 2.5 and 3.75 , respectively. This makes

$$
\mathfrak{R}\left(\sum_{l=1}^{2} \sum_{i=1}^{2} \widetilde{a}_{i}^{l}\right)=\mathfrak{R}\left(\sum_{l=1}^{2} \sum_{j=1}^{3} \tilde{b}_{j}^{l}\right)=122.25
$$

Also, $\mathfrak{R}\left(\sum_{h=1}^{2} \widetilde{e}_{h}\right)=104.5<122.25$. Therefore, insert a dummy conveyance having total fuzzy capacity equal to any fuzzy number with rank 17.75. As a dummy destination and a dummy conveyance are introduced, assume $\tilde{c}_{i 4 k}^{1}=\widetilde{c}_{i 4 k}^{2}=$ $\tilde{c}_{i j 3}^{1}=\tilde{c}_{i j 3}^{2}=(0,0,0,0)$ for all $i=1,2 ; j=1,2,3,4$; and $k=1,2,3$.

The considered problem is now balanced and consists of two objectives, two items, two sources, four destinations, and three different modes of transportation. The number of constraints is $2 \times 2+4 \times 2+3=15$.

Forming the crisp model by using the ranking technique as explained in Step 3.1, the model is as shown in the following.
TABLE 3: Unit penalties of transportation for item 1 in the second objective.

(a)

\begin{tabular}{lccc}
\hline \multicolumn{4}{c}{ Conveyance $k=1$} \\
Sources & $D_{1}$ & Destinations \\
& $(4,5,7,8)$ & $D_{2}$ & $D_{3}$ \\
\hline$S_{1}$ & $(6,8,9,11)$ & $(5,6,7,8)$ & $(7,9,10,12)$ \\
$S_{2}$ &
\end{tabular}

(b)

Conveyance $k=2$

\begin{tabular}{lccc} 
Sources & \multicolumn{3}{c}{ Destinations } \\
& $D_{1}$ & $D_{2}$ & $D_{3}$ \\
\hline$S_{1}$ & $(6,7,8,9)$ & $(4,6,7,9)$ & $(5,7,9,11)$ \\
$S_{2}$ & $(4,6,8,10)$ & $(7,9,11,13)$ & $(9,10,11,12)$ \\
\hline
\end{tabular}

TABLE 4: Unit penalties of transportation for item 2 in the second objective.

(a)

\begin{tabular}{lccc}
\hline \multicolumn{4}{c}{ Conveyance $k=1$} \\
Sources & $D_{1}$ & Destinations \\
& $(5,7,9,10)$ & $(4,6,7,9)$ & $D_{2}$ \\
\hline$S_{1}$ & $(10,11,13,14)$ & $(6,7,8,9)$ & $(7,9,11,12,13)$ \\
$S_{2}$ &
\end{tabular}

(b)

\begin{tabular}{lccc}
\hline & \multicolumn{3}{c}{ Conveyance $k=2$} \\
Sources & $D_{1}$ & Destinations & $D_{3}$ \\
\hline$S_{1}$ & $(7,8,9,10)$ & $(4,5,7,8)$ & $(8,10,11,12)$ \\
$S_{2}$ & $(6,8,10,12)$ & $(5,7,9,11)$ & $(9,10,12,14)$ \\
\hline
\end{tabular}

TABLE 5: Availability and demand data.

\begin{tabular}{lcc}
\hline Fuzzy availability & Fuzzy demand & Conveyance capacity \\
\hline$\tilde{a}_{1}^{1}=(21,24,26,28)$ & $\widetilde{b}_{1}^{1}=(14,16,19,22)$ & $\widetilde{e}_{1}=(46,49,51,53)$ \\
$\tilde{a}_{2}^{1}=(28,32,35,37)$ & $\widetilde{b}_{2}^{1}=(17,20,22,25)$ & $\widetilde{e}_{2}=(51,53,56,59)$ \\
$\widetilde{a}_{1}^{2}=(32,34,37,39)$ & $\widetilde{b}_{3}^{1}=(12,15,18,21)$ & \\
$\widetilde{a}_{2}^{2}=(25,28,30,33)$ & $\widetilde{b}_{1}^{2}=(20,23,25,28)$ & \\
& $\widetilde{b}_{2}^{2}=(16,18,19,22)$ & \\
& $\widetilde{b}_{3}^{2}=(15,17,19,21)$ & \\
\hline
\end{tabular}

Problem (P1)

$$
\text { Minimize } \quad \begin{aligned}
Z_{1}= & 8.25 x_{111}^{1}+12 x_{112}^{1}+0 x_{113}^{1}+7.5 x_{121}^{1} \\
& +9 x_{122}^{1}+0 x_{123}^{1}+13 x_{131}^{1}+10.5 x_{132}^{1} \\
& +0 x_{133}^{1}+0 x_{141}^{1}+0 x_{142}^{1}+0 x_{143}^{1} \\
& +11.5 x_{211}^{1}+12.25 x_{212}^{1}+0 x_{213}^{1} \\
& +7.5 x_{221}^{1}+9 x_{222}^{1}+0 x_{223}^{1}+14 x_{231}^{1}
\end{aligned}
$$




$$
\begin{aligned}
& +17 x_{232}^{1}+0 x_{233}^{1}+0 x_{241}^{1}+0 x_{242}^{1} \\
& +0 x_{243}^{1}+11 x_{111}^{2}+13.25 x_{112}^{2}+0 x_{113}^{2} \\
& +8.75 x_{121}^{2}+8.25 x_{122}^{2}+0 x_{123}^{2} \\
& +11.5 x_{131}^{2}+10.5 x_{132}^{2}+0 x_{133}^{2}+0 x_{141}^{2} \\
& +0 x_{142}^{2}+0 x_{143}^{2}+13.5 x_{211}^{2}+17 x_{212}^{2} \\
& +0 x_{213}^{2}+10.5 x_{221}^{2}+11.75 x_{222}^{2} \\
& +0 x_{223}^{2}+15 x_{231}^{2}+14.5 x_{232}^{2}+0 x_{233}^{2} \\
& +0 x_{241}^{2}+0 x_{242}^{2}+0 x_{243}^{2}
\end{aligned}
$$

Minimize $\quad Z_{2}=6 x_{111}^{1}+7.5 x_{112}^{1}+0 x_{113}^{1}+5.5 x_{121}^{1}$

$$
\begin{aligned}
& +6.5 x_{122}^{1}+0 x_{123}^{1}+9.5 x_{131}^{1} \\
& +8 x_{132}^{1}+0 x_{133}^{1} \\
& +0 x_{141}^{1}+0 x_{142}^{1}+0 x_{143}^{1}+8.5 x_{211}^{1} \\
& +7 x_{212}^{1}+0 x_{213}^{1}+6.5 x_{221}^{1}+10 x_{222}^{1} \\
& +0 x_{223}^{1}+8 x_{231}^{1}+10.5 x_{232}^{1}+0 x_{233}^{1} \\
& +0 x_{241}^{1}+0 x_{242}^{1}+0 x_{243}^{1}+7.75 x_{111}^{2} \\
& +8.5 x_{112}^{2}+0 x_{113}^{2}+6.5 x_{121}^{2}+6 x_{122}^{2} \\
& +0 x_{123}^{2}+11.25 x_{131}^{2}+10.25 x_{132}^{2} \\
& +0 x_{133}^{2}+0 x_{141}^{2}+0 x_{142}^{2}+0 x_{143}^{2}+12 x_{211}^{2} \\
& +9 x_{212}^{2}+0 x_{213}^{2}+7.5 x_{221}^{2}+8 x_{222}^{2} \\
& +0 x_{223}^{2}+9.75 x_{231}^{2}+11.25 x_{232}^{2}+0 x_{233}^{2} \\
& +0 x_{241}^{2}+0 x_{242}^{2}+0 x_{243}^{2}
\end{aligned}
$$

subject to $x_{111}^{1}+x_{112}^{1}+x_{113}^{1}+x_{121}^{1}+x_{122}^{1}$

$$
\begin{aligned}
& +x_{123}^{1}+x_{131}^{1}+x_{132}^{1}+x_{133}^{1} \\
& +x_{141}^{1}+x_{142}^{1}+x_{143}^{1}=24.75 \\
& x_{111}^{2}+x_{112}^{2}+x_{113}^{2}+x_{121}^{2}+x_{122}^{2} \\
& +x_{123}^{2}+x_{131}^{2}+x_{132}^{2}+x_{133}^{2} \\
& +x_{141}^{2}+x_{142}^{2}+x_{143}^{2}=35.5 \\
& x_{211}^{1}+x_{212}^{1}+x_{213}^{1}+x_{221}^{1}+x_{222}^{1} \\
& +x_{223}^{1}+x_{231}^{1}+x_{232}^{1}+x_{233}^{1} \\
& +x_{241}^{1}+x_{242}^{1}+x_{243}^{1}=33.0 \\
& x_{211}^{2}+x_{212}^{2}+x_{213}^{2}+x_{221}^{2}+x_{222}^{2}
\end{aligned}
$$

$$
\begin{aligned}
& +x_{223}^{2}+x_{231}^{2}+x_{232}^{2}+x_{233}^{2} \\
& +x_{241}^{2}+x_{242}^{2}+x_{243}^{2}=29.0 \\
& x_{111}^{1}+x_{112}^{1}+x_{113}^{1}+x_{211}^{1} \\
& +x_{212}^{1}+x_{213}^{1}=17.75 \\
& x_{111}^{2}+x_{112}^{2}+x_{113}^{2}+x_{211}^{2} \\
& +x_{212}^{2}+x_{213}^{2}=24.0 \\
& x_{121}^{1}+x_{122}^{1}+x_{123}^{1}+x_{221}^{1}+x_{222}^{1}+x_{223}^{1}=21.0 \\
& x_{121}^{2}+x_{122}^{2}+x_{123}^{2}+x_{221}^{2}+x_{222}^{2} \\
& +x_{223}^{2}=18.75 \\
& x_{131}^{1}+x_{132}^{1}+x_{133}^{1}+x_{231}^{1}+x_{232}^{1}+x_{233}^{1}=16.5 \\
& x_{131}^{2}+x_{132}^{2}+x_{133}^{2}+x_{231}^{2}+x_{232}^{2}+x_{233}^{2}=18.0 \\
& x_{141}^{1}+x_{142}^{1}+x_{143}^{1}+x_{241}^{1}+x_{242}^{1}+x_{243}^{1}=2.5 \\
& x_{141}^{2}+x_{142}^{2}+x_{143}^{2}+x_{241}^{2} \\
& +x_{242}^{2}+x_{243}^{2}=3.75 \\
& x_{111}^{1}+x_{121}^{1}+x_{131}^{1}+x_{141}^{1}+x_{211}^{1} \\
& +x_{221}^{1}+x_{231}^{1}+x_{241}^{1} \\
& +x_{111}^{2}+x_{121}^{2}+x_{131}^{2}+x_{141}^{2}+x_{211}^{2} \\
& +x_{221}^{2}+x_{231}^{2}+x_{241}^{2}=49.75 \\
& x_{112}^{1}+x_{122}^{1}+x_{132}^{1}+x_{142}^{1}+x_{212}^{1}+x_{222}^{1} \\
& +x_{232}^{1}+x_{242}^{1}+x_{112}^{2}+x_{122}^{2}+x_{132}^{2}+x_{142}^{2} \\
& +x_{212}^{2}+x_{222}^{2}+x_{232}^{2}+x_{242}^{2}=54.75 \\
& x_{113}^{1}+x_{123}^{1}+x_{133}^{1}+x_{143}^{1}+x_{213}^{1}+x_{223}^{1} \\
& +x_{233}^{1}+x_{243}^{1}+x_{113}^{2}+x_{123}^{2}+x_{133}^{2}+x_{143}^{2} \\
& 2 \\
& 2 \\
& 27.75 . \\
& \hline
\end{aligned}
$$

Applying the fuzzy programming technique [4] to the above crisp multiobjective linear programming problem, the results obtained are as follows:

$$
\begin{aligned}
& L_{1}\left(\min \left(Z_{1}\right)\right)=938.06, \quad U_{1}\left(\max \left(Z_{1}\right)\right)=1061.0, \\
& L_{2}\left(\min \left(Z_{2}\right)\right)=682.5, \quad U_{2}\left(\max \left(Z_{2}\right)\right)=777.875,
\end{aligned}
$$


and the obtained optimal compromise solution is given as follows:

$$
\begin{array}{lll}
x_{111}^{1}=8.25, & x_{132}^{1}=16.5, & x_{212}^{1}=9.5, \\
x_{221}^{1}=21.0, & x_{242}^{1}=2.5, & x_{111}^{2}=18.63, \\
x_{122}^{2}=16.88, & x_{212}^{2}=1.79, & x_{213}^{2}=3.58, \\
x_{221}^{1}=1.88, & x_{232}^{2}=3.83, & x_{233}^{2}=14.17, \\
x_{242}^{2}=3.75, & \lambda=0.78, & Z_{1}=945.3425 ; \\
& Z_{2}=703.46 .
\end{array}
$$

Forming the crisp model using the concept of minimum of fuzzy number as explained in Step 3.2 of the proposed method taking $M=500, \lambda_{1}=\lambda_{2}=0.4, \lambda_{3}=0.2$, the following crisp multiobjective transportation problem is obtained.

Problem (P2)

$$
\text { Minimize } \quad \begin{aligned}
Z_{1}= & 2.9 x_{111}^{1}+4.4 x_{112}^{1}+0 x_{113}^{1} \\
& +2.5 x_{121}^{1}+3.2 x_{122}^{1}+0 x_{123}^{1} \\
& +4.8 x_{131}^{1}+3.7 x_{132}^{1}+0 x_{133}^{1}+0 x_{141}^{1} \\
& +0 x_{142}^{1}+0 x_{143}^{1}+4.1 x_{211}^{1}+4.6 x_{212}^{1} \\
& +0 x_{213}^{1}+2.8 x_{221}^{1}+3.2 x_{222}^{1}+0 x_{223}^{1} \\
& +5.2 x_{231}^{1}+6.4 x_{232}^{1}+0 x_{233}^{1}+0 x_{241}^{1} \\
& +0 x_{242}^{1}+0 x_{243}^{1}+4.1 x_{111}^{2}+5 x_{112}^{2} \\
& +0 x_{113}^{2}+3 x_{121}^{2}+3 x_{122}^{2}+0 x_{123}^{2} \\
& +4.4 x_{131}^{2}+3.9 x_{132}^{2}+0 x_{133}^{2}+0 x_{141}^{2} \\
& +0 x_{142}^{2}+0 x_{143}^{2}+5.1 x_{211}^{2}+6.4 x_{212}^{2} \\
& +0 x_{213}^{2}+3.7 x_{221}^{2}+4.3 x_{222}^{2}+0 x_{223}^{2} \\
& +5.6 x_{231}^{2}+5.6 x_{232}^{2}+0 x_{233}^{2}+0 x_{241}^{2} \\
& +0 x_{242}^{2}+0 x_{243}^{2}+200
\end{aligned}
$$

Minimize $\quad Z_{2}=2.1 x_{111}^{1}+2.8 x_{112}^{1}+0 x_{113}^{1}+1.9 x_{121}^{1}$

$$
\begin{aligned}
& +2.3 x_{122}^{1}+0 x_{123}^{1}+3.5 x_{131}^{1}+2.8 x_{132}^{1} \\
& +0 x_{133}^{1}+0 x_{141}^{1}+0 x_{142}^{1}+0 x_{143}^{1} \\
& +3.1 x_{211}^{1}+2.4 x_{212}^{1}+0 x_{213}^{1}+2.4 x_{221}^{1} \\
& +3.6 x_{222}^{1}+0 x_{223}^{1}+2.9 x_{231}^{1}+4 x_{232}^{1} \\
& +0 x_{233}^{1}+0 x_{241}^{1}+0 x_{242}^{1}+0 x_{243}^{1} \\
& +2.7 x_{111}^{2}+3.2 x_{112}^{2}+0 x_{113}^{2}+2.3 x_{121}^{2}
\end{aligned}
$$

$$
\begin{aligned}
& +2.1 x_{122}^{2}+0 x_{123}^{2}+4.2 x_{131}^{2}+3.8 x_{132}^{2} \\
& +0 x_{133}^{2}+0 x_{141}^{2}+0 x_{142}^{2}+0 x_{143}^{2}+4.5 x_{211}^{2} \\
& +3.2 x_{212}^{2}+0 x_{213}^{2}+2.8 x_{221}^{2}+2.8 x_{222}^{2} \\
& +0 x_{223}^{2}+3.5 x_{231}^{2}+4.2 x_{232}^{2} \\
& +0 x_{233}^{2}+0 x_{241}^{2}+0 x_{242}^{2}+0 x_{243}^{2}+200
\end{aligned}
$$

subject to the constraints as in Problem (P1).

Solving this problem using fuzzy programming technique, obtained results are as follows:

$$
\begin{array}{cc}
L_{1}\left(\min \left(Z_{1}\right)\right)=540.88, & A_{L}\left(Z_{1}\right)=170.25, \\
C\left(Z_{1}\right)=936.625, & A_{R}\left(Z_{1}\right)=174.625 \\
U_{1}\left(\min \left(Z_{1}\right)\right)=587.75, & A_{L}\left(Z_{1}\right)=181.75, \\
C\left(Z_{1}\right)=1051.125, & A_{R}\left(Z_{1}\right)=200.0 \\
L_{2}\left(\min \left(Z_{2}\right)\right)=439.05, & A_{L}\left(Z_{2}\right)=170.25, \\
C\left(Z_{2}\right)=733.875, & A_{R}\left(Z_{2}\right)=161.75 . \\
U_{2}\left(\min \left(Z_{2}\right)\right)=476.3, & A_{L}\left(Z_{2}\right)=144.5, \\
C\left(Z_{2}\right)=768.375, & A_{R}\left(Z_{2}\right)=133.75
\end{array}
$$

The optimal compromise solution is found to be

$$
\begin{array}{ccc}
x_{111}^{1}=8.25, & x_{132}^{1}=16.5, & x_{212}^{1}=9.5, \\
x_{221}^{1}=21.0, & x_{242}^{1}=2.5, & x_{111}^{2}=20.5, \\
x_{122}^{2}=12.56, & x_{132}^{2}=2.44, & x_{212}^{2}=1.31, \\
x_{213}^{2}=2.19, & x_{222}^{2}=6.19, \quad x_{233}^{2}=15.56, \\
x_{242}^{2}=3.75, \quad \lambda=0.73 .
\end{array}
$$

$Z_{1}=553.722, A_{L}\left(Z_{1}\right)=161.25, C\left(Z_{1}\right)=963.025, A_{R}\left(Z_{1}\right)$ $=165.06$, and $[872.37,1053.68]$ is the core of the objective function $Z_{1}$.

$$
Z_{2}=449.247, A_{L}\left(Z_{2}\right)=163.815, C\left(Z_{2}\right)=710.79,
$$
$A_{R}\left(Z_{1}\right)=152.405$, and $[624.26,797.32]$ is the core of the objective function $Z_{2}$.

6.2. Example 2. If a MOMISTP in which availability of one or more items is less than the corresponding demand and/or total conveyance capacity is less than total demand is solved by the method presented in [19], it terminates with the conclusion that the problem is infeasible. However, such problems can be solved by the method proposed in this paper, since it starts after balancing the unbalanced problem. Let the availability data in Example 1 (Table 5) be changed to Table 6, so that the total availability of some items is less and of some items is more than their total demand, keeping the demand and the unit penalty of transportation the same. 
TABLE 6: Availability and demand data.

\begin{tabular}{lcc}
\hline Fuzzy availability & Fuzzy demand & Conveyance capacity \\
\hline$\widetilde{a}_{1}^{1}=(15,18,22,27)$ & $\widetilde{b}_{1}^{1}=(14,16,19,22)$ & $\widetilde{e}_{1}=(46,49,51,53)$ \\
$\widetilde{a}_{2}^{1}=(20,25,29,32)$ & $\widetilde{b}_{2}^{1}=(17,20,22,25)$ & $\widetilde{e}_{2}=(51,53,56,59)$ \\
$\tilde{a}_{1}^{2}=(32,34,37,39)$ & $\widetilde{b}_{3}^{1}=(12,15,18,21)$ & \\
$\tilde{a}_{2}^{2}=(25,28,30,33)$ & $\widetilde{b}_{1}^{2}=(20,23,25,28)$ & \\
& $\widetilde{b}_{2}^{2}=(16,18,19,22)$ & \\
& $\widetilde{b}_{3}^{2}=(15,17,19,21)$ & \\
\hline
\end{tabular}

We find that

$$
\begin{gathered}
\Re\left(\sum_{i=1}^{2} \widetilde{a}_{i}^{1}\right)=47<\mathfrak{R}\left(\sum_{j=1}^{3} \widetilde{b}_{j}^{1}\right)=55.25, \\
\mathfrak{R}\left(\sum_{i=1}^{2} \widetilde{a}_{i}^{2}\right)=64.5>\mathfrak{R}\left(\sum_{j=1}^{3} \widetilde{b}_{j}^{2}\right)=60.75, \\
\Re\left(\sum_{h=1}^{2} \widetilde{e}_{h}\right)=104.5 .
\end{gathered}
$$

So to balance the problem add a dummy source $S_{3}$ having availability of item 1 only and a dummy destination $D_{4}$ with demand of item 2 only, equal to any nonnegative fuzzy numbers whose ranks are 8.25 and 3.75 , respectively, and a dummy conveyance having availability equal to any fuzzy number having rank 15.25. Assume the unit transportation penalties from $S_{3}$ to all the destinations and from all the sources to $D_{4}$ via any of the conveyances as $\widetilde{c}_{3 j k}^{1}=\widetilde{c}_{i 4 k}^{2}=\widetilde{c}_{i j 3}^{1}=$ $\tilde{c}_{i j 3}^{2}=(0,0,0,0)$ for all $i=1,2,3 ; j=1,2,3,4$; and $k=1,2,3$.

After balancing the problem and forming the expected value model using ranking technique the obtained problem is shown as follows.

\section{Problem (P3)}

$$
\text { Minimize } \quad \begin{aligned}
Z_{1}= & 8 \\
+ & +5 x_{111}^{1}+12 x_{112}^{1}+0 x_{113}^{1} \\
& +10.5 x_{132}^{1}+9 x_{122}^{1}+0 x_{123}^{1}+13 x_{131}^{1} \\
& +0 x_{213}^{1}+7.5 x_{221}^{1}+9 x_{222}^{1}+0 x_{223}^{1} \\
& +14 x_{231}^{1}+17 x_{232}^{1}+0 x_{233}^{1}+0 x_{311}^{1} \\
& +0 x_{312}^{1}+0 x_{313}^{1}+0 x_{321}^{1}+0 x_{322}^{1} \\
& +0 x_{323}^{1}+0 x_{331}^{1}+0 x_{332}^{1}+0 x_{333}^{1}+11 x_{111}^{2} \\
& +13.25 x_{112}^{2}+0 x_{113}^{2}+8.75 x_{121}^{2}+8.25 x_{122}^{2} \\
& +0 x_{123}^{2}+11.5 x_{131}^{2}+10.5 x_{132}^{2}+0 x_{133}^{2} \\
& +0 x_{141}^{2}+0 x_{142}^{2}+0 x_{143}^{2}+13.5 x_{211}^{2} \\
& +17 x_{212}^{2}
\end{aligned}
$$

$$
\begin{aligned}
& +0 x_{213}^{2}+10.5 x_{221}^{2}+11.75 x_{222}^{2}+0 x_{223}^{2} \\
& +15 x_{231}^{2}+14.5 x_{232}^{2}+0 x_{233}^{2} \\
& +0 x_{241}^{2}+0 x_{242}^{2}+0 x_{243}^{2}
\end{aligned}
$$

Minimize

$$
\begin{aligned}
Z_{2}= & 6 x_{111}^{1}+7.5 x_{112}^{1}+0 x_{113}^{1}+5.5 x_{121}^{1} \\
& +6.5 x_{122}^{1}+0 x_{123}^{1}+9.5 x_{131}^{1}+8 x_{132}^{1} \\
& +0 x_{133}^{1}+8.5 x_{211}^{1}+7 x_{212}^{1}+0 x_{213}^{1} \\
& +6.5 x_{221}^{1}+10 x_{222}^{1}+0 x_{223}^{1}+8 x_{231}^{1} \\
& +10.5 x_{232}^{1}+0 x_{233}^{1}+0 x_{311}^{1}+0 x_{312}^{1} \\
& +0 x_{313}^{1}+0 x_{321}^{1}+0 x_{322}^{1}+0 x_{323}^{1} \\
& +0 x_{331}^{1}+0 x_{332}^{1}+0 x_{333}^{1}+7.75 x_{111}^{2} \\
& +8.5 x_{112}^{2}+0 x_{113}^{2}+6.5 x_{121}^{2}+6 x_{122}^{2} \\
& +0 x_{123}^{2}+11.25 x_{131}^{2}+10.25 x_{132}^{2}+0 x_{133}^{2} \\
& +0 x_{141}^{2}+0 x_{142}^{2}+0 x_{143}^{2}+12 x_{211}^{2}+9 x_{212}^{2} \\
& +0 x_{213}^{2}+7.5 x_{221}^{2}+8 x_{222}^{2}+0 x_{223}^{2} \\
& +9.75 x_{231}^{2}+11.25 x_{232}^{2}+0 x_{233}^{2} \\
& +0 x_{241}^{2}+0 x_{242}^{2}+0 x_{243}^{2}
\end{aligned}
$$

subject to $x_{111}^{1}+x_{112}^{1}+x_{113}^{1}+x_{121}^{1}+x_{122}^{1}$

$$
\begin{aligned}
& +x_{123}^{1}+x_{131}^{1}+x_{132}^{1}+x_{133}^{1}=20.5 \\
& x_{111}^{2}+x_{112}^{2}+x_{113}^{2}+x_{121}^{2}+x_{122}^{2} \\
& +x_{123}^{2}+x_{131}^{2}+x_{132}^{2}+x_{133}^{2} \\
& +x_{141}^{2}+x_{142}^{2}+x_{143}^{2}=35.5 \\
& x_{211}^{1}+x_{212}^{1}+x_{213}^{1}+x_{221}^{1}+x_{222}^{1} \\
& +x_{223}^{1}+x_{231}^{1}+x_{232}^{1}+x_{233}^{1}=26.5 \\
& x_{211}^{2}+x_{212}^{2}+x_{213}^{2}+x_{221}^{2}+x_{222}^{2}+x_{223}^{2} \\
& +x_{231}^{2}+x_{232}^{2}+x_{233}^{2}+x_{241}^{2} \\
& +x_{242}^{2}+x_{243}^{2}=29.0 \\
& x_{311}^{1}+x_{312}^{1}+x_{313}^{1}+x_{321}^{1}+x_{322}^{1} \\
& +x_{323}^{1}+x_{331}^{1}+x_{332}^{1}+x_{333}^{1}=8.25 \\
& x_{111}^{1}+x_{112}^{1}+x_{113}^{1}+x_{211}^{1}+x_{212}^{1} \\
& +x_{213}^{1}+x_{311}^{1}+x_{312}^{1}+x_{313}^{1}=17.75 \\
& x_{111}^{2}+x_{112}^{2}+x_{113}^{2}+x_{211}^{2}+x_{212}^{2}+x_{213}^{2}=24.0
\end{aligned}
$$




$$
\begin{aligned}
& x_{121}^{1}+x_{122}^{1}+x_{123}^{1}+x_{221}^{1}+x_{222}^{1} \\
& +x_{223}^{1}+x_{321}^{1}+x_{322}^{1}+x_{323}^{1}=21.0 \\
& x_{121}^{2}+x_{122}^{2}+x_{123}^{2}+x_{221}^{2}+x_{222}^{2}+x_{223}^{2}=18.75 \\
& x_{131}^{1}+x_{132}^{1}+x_{133}^{1}+x_{231}^{1} \\
& +x_{232}^{1}+x_{233}^{1}+x_{331}^{1}+x_{332}^{1}+x_{333}^{1}=16.5 \\
& x_{131}^{2}+x_{132}^{2}+x_{133}^{2}+x_{231}^{2}+x_{232}^{2}+x_{233}^{2}=18.0 \\
& x_{141}^{2}+x_{142}^{2}+x_{143}^{2}+x_{241}^{2}+x_{242}^{2}+x_{243}^{2}=3.75 \\
& x_{111}^{1}+x_{121}^{1}+x_{131}^{1}+x_{211}^{1}+x_{221}^{1} \\
& +x_{231}^{1}+x_{311}^{1}+x_{321}^{1}+x_{331}^{1} \\
& +x_{111}^{2}+x_{121}^{2}+x_{131}^{2}+x_{141}^{2} \\
& +x_{211}^{2}+x_{221}^{2}+x_{231}^{2}+x_{241}^{2}=49.75 \\
& x_{112}^{1}+x_{122}^{1}+x_{132}^{1}+x_{212}^{1}+x_{222}^{1} \\
& +x_{232}^{1}+x_{312}^{1}+x_{322}^{1}+x_{332}^{1} \\
& +x_{112}^{2}+x_{122}^{2}+x_{132}^{2}+x_{142}^{2} \\
& +x_{212}^{2}+x_{222}^{2}+x_{232}^{2}+x_{242}^{2}=54.75 \\
& x_{113}^{1}+x_{123}^{1}+x_{133}^{1}+x_{213}^{1}+x_{223}^{1} \\
& +x_{233}^{1}+x_{313}^{1}+x_{323}^{1}+x_{333}^{1} \\
& +x_{113}^{2}+x_{123}^{2}+x_{133}^{2}+x_{143}^{2} \\
& 2 \\
& 2 \\
& 2
\end{aligned}
$$

Applying the fuzzy programming technique, we obtain

$$
\begin{array}{ll}
L_{1}=872.81, & U_{1}=993.875, \\
L_{2}=640.63, & U_{2}=728.125 .
\end{array}
$$

The optimal compromise solution is

$$
\begin{array}{ccc}
\lambda=0.74, & x_{111}^{1}=12.25, & x_{132}^{1}=8.25, \\
x_{212}^{1}=5.5, & x_{221}^{1}=21, & x_{332}^{1}=8.25, \\
x_{111}^{2}=16.5, & x_{122}^{2}=18.75, & x_{132}^{2}=0.25, \\
x_{212}^{2}=3.03, & x_{213}^{2}=4.47, & x_{232}^{2}=6.97, \\
x_{233}^{2}=10.78, & x_{242}^{2}=3.75, & Z_{1}=903.95, \\
& Z_{2}=663.12 . &
\end{array}
$$

Converting the same problem to crisp problem using the concept of minimum of fuzzy number, the model obtained is as follows.

\section{Problem (P4)}

Minimize $\quad Z_{1}=2.9 x_{111}^{1}+4.4 x_{112}^{1}+0 x_{113}^{1}+2.5 x_{121}^{1}$

$$
\begin{aligned}
& +3.2 x_{122}^{1}+0 x_{123}^{1}+4.8 x_{131}^{1}+3.7 x_{132}^{1} \\
& +0 x_{133}^{1}+4.1 x_{211}^{1}+4.6 x_{212}^{1}+0 x_{213}^{1} \\
& +2.8 x_{221}^{1}+3.2 x_{222}^{1}+0 x_{223}^{1}+5.2 x_{231}^{1} \\
& +6.4 x_{232}^{1}+0 x_{233}^{1}+0 x_{311}^{1}+0 x_{312}^{1}+0 x_{313}^{1} \\
& +0 x_{321}^{1}+0 x_{322}^{1}+0 x_{323}^{1}+0 x_{331}^{1} \\
& +0 x_{332}^{1}+0 x_{333}^{1}+4.1 x_{111}^{2}+5 x_{112}^{2} \\
& +0 x_{113}^{2}+3 x_{121}^{2}+3 x_{122}^{2}+0 x_{123}^{2} \\
& +4.4 x_{131}^{2}+3.9 x_{132}^{2}+0 x_{133}^{2}+0 x_{141}^{2} \\
& +0 x_{142}^{2}+0 x_{143}^{2}+5.1 x_{211}^{2}+6.4 x_{212}^{2} \\
& +0 x_{213}^{2}+3.7 x_{221}^{2}+4.3 x_{222}^{2}+0 x_{223}^{2} \\
& +5.6 x_{231}^{2}+5.6 x_{232}^{2}+0 x_{233}^{2} \\
& +0 x_{241}^{2}+0 x_{242}^{2}+0 x_{243}^{2}+200
\end{aligned}
$$

Minimize $\quad Z_{1}=2.1 x_{111}^{1}+2.8 x_{112}^{1}+0 x_{113}^{1}+1.9 x_{121}^{1}$

$$
\begin{aligned}
& +2.3 x_{122}^{1}+0 x_{123}^{1}+3.5 x_{131}^{1}+2.8 x_{132}^{1} \\
& +0 x_{133}^{1}+3.1 x_{211}^{1}+2.4 x_{212}^{1}+0 x_{213}^{1} \\
& +2.4 x_{221}^{1}+3.6 x_{222}^{1}+0 x_{223}^{1}+2.9 x_{231}^{1} \\
& +4 x_{232}^{1}+0 x_{233}^{1}+0 x_{311}^{1}+0 x_{312}^{1} \\
& +0 x_{313}^{1}+0 x_{321}^{1}+0 x_{322}^{1}+0 x_{323}^{1}+0 x_{331}^{1} \\
& +0 x_{332}^{1}+0 x_{333}^{1}+2.7 x_{111}^{2}+3.2 x_{112}^{2} \\
& +0 x_{113}^{2}+2.3 x_{121}^{2}+2.1 x_{122}^{2}+0 x_{123}^{2} \\
& +4.2 x_{131}^{2}+3.8 x_{132}^{2}+0 x_{133}^{2}+0 x_{141}^{2} \\
& +0 x_{142}^{2}+0 x_{143}^{2}+4.5 x_{211}^{2}+3.2 x_{212}^{2} \\
& +0 x_{213}^{2}+2.8 x_{221}^{2}+2.8 x_{222}^{2}+0 x_{223}^{2} \\
& +3.5 x_{231}^{2}+4.2 x_{232}^{2}+0 x_{233}^{2}+0 x_{241}^{2} \\
& +0 x_{242}^{2}+0 x_{243}^{2}+200
\end{aligned}
$$

subject to the constraints as in Problem (P3).

Solving this problem using fuzzy programming technique, the obtained results are as follows:

$$
\begin{gathered}
L_{1}\left(\min \left(Z_{1}\right)\right)=519.05, \quad A_{L}\left(Z_{1}\right)=150.375, \\
C\left(Z_{1}\right)=871.75, \quad A_{R}\left(Z_{1}\right)=152.5
\end{gathered}
$$




$$
\begin{array}{cc}
U_{1}\left(\min \left(Z_{1}\right)\right)=563.925, & A_{L}\left(Z_{1}\right)=168.125, \\
C\left(Z_{1}\right)=984.75, & A_{R}\left(Z_{1}\right)=186.375 \\
L_{2}\left(\min \left(Z_{2}\right)\right)=424.10, & A_{L}\left(Z_{2}\right)=160.75 \\
C\left(Z_{2}\right)=645.5, & A_{R}\left(Z_{2}\right)=151 \\
U_{2}\left(\min \left(Z_{2}\right)\right)=463.20, & A_{L}\left(Z_{2}\right)=131.75 \\
C\left(Z_{2}\right)=728.625, & A_{R}\left(Z_{2}\right)=122.25 .
\end{array}
$$

The optimal compromise solution is found to be

$$
\begin{array}{ccc}
x_{111}^{1}=12.25, & x_{132}^{1}=8.25, & x_{212}^{1}=5.5, \\
x_{221}^{1}=21.0, & x_{332}^{1}=8.25, & x_{111}^{2}=16.5, \\
x_{122}^{2}=13.55, & x_{132}^{2}=5.45, & x_{212}^{2}=4.8, \\
x_{213}^{2}=2.7, & x_{222}^{2}=5.2, & x_{233}^{2}=12.55, \\
& x_{242}^{2}=3.75 .
\end{array}
$$

$Z_{1}=532.785, A_{L}\left(Z_{1}\right)=147.625, C\left(Z_{1}\right)=905.375, A_{R}\left(Z_{1}\right)$ $=148.425$, and $[828.1,982.65]$ is the core of the objective function $Z_{1}$.

$Z_{2}=436.06, A_{L}\left(Z_{2}\right)=148.375, C\left(Z_{2}\right)=669.825$, $A_{R}\left(Z_{1}\right)=137.4$, and $[590.55,749.1]$ is the core of the objective function $Z_{2}$.

Similarly, other problems, in which availability of all the items is less/more than its demand and/or the total conveyance capacity is less/more than the total availability or total demand, can be solved by the proposed method.

\section{Results}

The above models are solved with the help of MAPPLE software. Kundu et al. [19] obtained the solution of the MOMISTP considered in Section 6.1 using the defuzzification technique given by Kikuchi [24]. The results for both problems are shown in Tables 7 and 8 and are interpreted in Section 8.

\section{Interpretation of Results}

The objective values found using the concept of minimum of fuzzy numbers for Example 1 (Section 6.1) can be shown by Figures 2 and 3.

From the membership function of the objective function $Z_{1}$ shown in Figure 2, the following information about the minimum transportation cost for the objective function $Z_{1}$ may be interpreted.

(i) According to the decision maker minimum transportation cost for the transportation will be greater than 731.18 units and will be less than 1202.49 units.

(ii) The maximum chances are that the minimum transportation cost will lie in the range 872.37-1053.68 units.

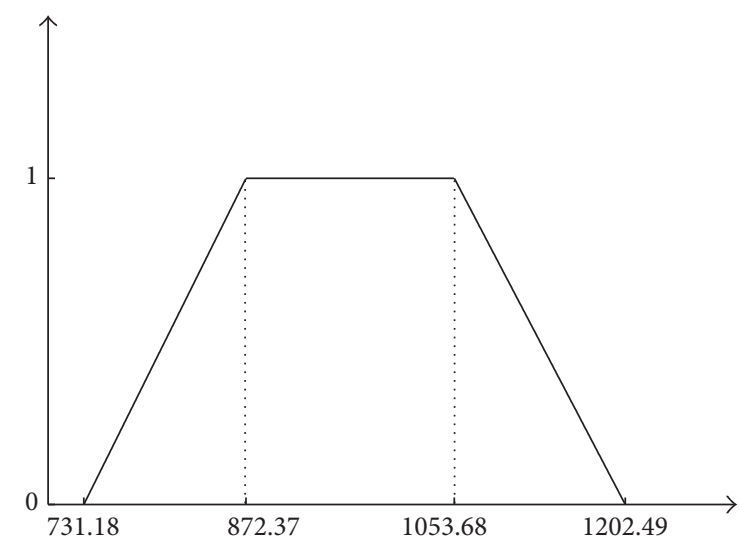

Figure 2: Optimal value of $Z_{1}$.

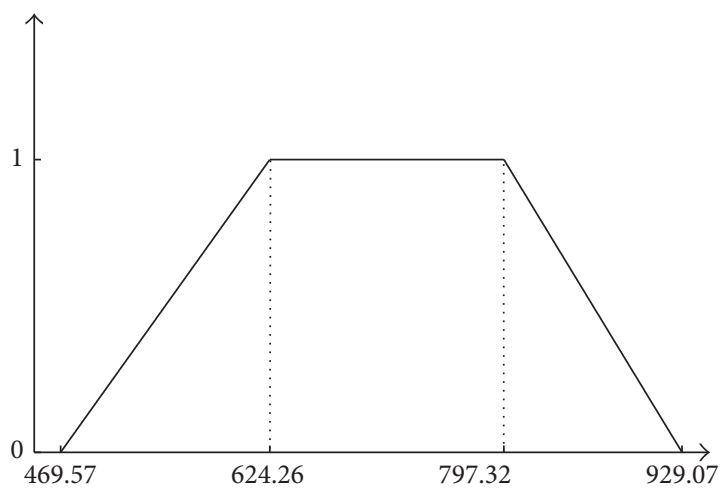

Figure 3: Optimal value of $Z_{2}$.

(iii) The overall level of satisfaction for other values of the minimum transportation cost (say $x$ ) is $\mu_{\widetilde{Z}_{1}}(x) \times 100$, where

$$
\mu_{\widetilde{Z}_{1}}(x)= \begin{cases}\frac{(x-731.18)}{141.19}, & 731.18 \leq x<872.37 \\ 1, & 872.37 \leq x \leq 1053.68 \\ \frac{(1202.49-x)}{148.81}, & 1053.68<x \leq 1202.49 \\ 0, & \text { otherwise. }\end{cases}
$$

Similarly, the results about the minimum transportation cost for the objective function $Z_{2}$ can be interpreted. The decision maker has more information about the objective function using this concept. It has been found that the objective values obtained using the rank of fuzzy numbers $Z_{1}=945.3425$ and $Z_{2}=703.46$ lie within the cores $[872.37,1053.68]$ and $[624.26$, $797.32]$ of the objective functions $Z_{1}$ and $Z_{2}$, respectively, and are close to their centre of cores.

The results of Example 2 (Section 6.2) can also be interpreted similarly.

\section{Conclusions}

Our results show that, unlike [19], the expected value model gives the feasible and hence the optimal solution of Example 1 
TABLE 7: Results using the existing method [19].

\begin{tabular}{|c|c|c|c|}
\hline Example & Optimal compromise solution & $Z_{1}$ & $Z_{2}$ \\
\hline \multicolumn{4}{|c|}{ Ranking method } \\
\hline 1 & $\begin{array}{l}x_{111}^{1}=3.1686, x_{121}^{1}=5.8313, x_{221}^{1}=13.8686, x_{132}^{1}=14.7 \\
x_{212}^{1}=12.6314, x_{111}^{2}=22.7, x_{221}^{2}=5.6314, x_{122}^{2}=11.1 \\
x_{222}^{2}=1.0686, x_{232}^{2}=16.8, \lambda=0.6989\end{array}$ & 1139.536 & 837.4808 \\
\hline 2 & Infeasible solution & - & - \\
\hline \multicolumn{4}{|c|}{ Minimum of fuzzy number } \\
\hline 1 & $\begin{array}{l}x_{111}^{1}=9, x_{221}^{1}=19.7, x_{132}^{1}=14.7, x_{212}^{1}=6.8 \\
x_{111}^{2}=18.3839, x_{231}^{2}=4.1160, x_{122}^{2}=2.7321 \\
x_{132}^{2}=12.68394, x_{212}^{2}=4.3160, x_{222}^{2}=15.0678, \lambda=0.753\end{array}$ & $\begin{array}{c}Z_{1}=615.4325 \\
\quad \text { core of } Z_{1}= \\
{[1039.232,1227.548]}\end{array}$ & $\begin{array}{c}Z_{2}=497.6407 \\
\quad \text { core of } Z_{2}= \\
{[753.1357,935.7518]}\end{array}$ \\
\hline 2 & Infeasible solution & - & - \\
\hline
\end{tabular}

TABLE 8: Results using the proposed method.

\begin{tabular}{|c|c|c|c|}
\hline Example & Optimal compromise solution & $Z_{1}$ & $Z_{2}$ \\
\hline \multicolumn{4}{|c|}{ Ranking method } \\
\hline 1 & $\begin{array}{l}x_{111}^{1}=8.25, x_{132}^{1}=16.5, x_{212}^{1}=9.5, x_{221}^{1}=21.0, x_{242}^{1}=2.5 \\
x_{111}^{2}=18.63, x_{122}^{2}=16.88, x_{212}^{2}=1.79, x_{213}^{2}=3.58 \\
x_{221}^{1}=1.88, x_{232}^{2}=3.83, x_{233}^{2}=14.17, x_{242}^{2}=3.75, \lambda=0.78\end{array}$ & 945.34 & 703.46 \\
\hline 2 & $\begin{array}{l}x_{111}^{1}=12.25, x_{132}^{1}=8.25, x_{212}^{1}=5.5, x_{221}^{1}=21, x_{332}^{1}=8.25 \\
x_{111}^{2}=16.5, x_{122}^{2}=18.75, x_{132}^{2}=0.25, x_{212}^{2}=3.03 \\
x_{213}^{2}=4.47, x_{232}^{2}=6.97, x_{233}^{2}=10.78, x_{242}^{2}=3.75, \lambda=0.74\end{array}$ & 903.95 & 663.12 \\
\hline \multicolumn{4}{|c|}{ Minimum of fuzzy number } \\
\hline 1 & $\begin{array}{l}x_{111}^{1}=8.25, x_{132}^{1}=16.5, x_{212}^{1}=9.5, x_{221}^{1}=21.0, x_{242}^{1}=2.5 \\
x_{111}^{2}=20.5, x_{122}^{2}=12.56, x_{132}^{2}=2.44, x_{212}^{2}=1.31 \\
x_{213}^{2}=2.19, x_{222}^{2}=6.19, x_{233}^{2}=15.56, x_{242}^{2}=3.75, \lambda=0.73\end{array}$ & $\begin{array}{c}Z_{1}=553.722 \\
\text { core of } Z_{1}=[872.37,1053.68]\end{array}$ & $\begin{aligned} Z_{2} & =449.247 \\
\text { core of } Z_{2} & =[624.26,797.32]\end{aligned}$ \\
\hline 2 & $\begin{array}{l}x_{111}^{1}=12.25, x_{132}^{1}=8.25, x_{212}^{1}=5.5, x_{221}^{1}=21.0 \\
x_{332}^{1}=8.25, x_{111}^{2}=16.5, x_{122}^{2}=13.55, x_{132}^{2}=5.45 \\
x_{212}^{2}=4.8, x_{213}^{2}=2.7, x_{222}^{2}=5.2, x_{233}^{2}=12.55, x_{242}^{2}=3.75\end{array}$ & $\begin{aligned} Z_{1} & =532.785 \\
\text { core of } Z_{1} & =[828.1,982.65]\end{aligned}$ & $\begin{aligned} Z_{2} & =436.06 \\
\text { core of } Z_{2} & =[590.55,749.1]\end{aligned}$ \\
\hline
\end{tabular}

(Section 6.1). It has been found that the obtained optimal compromise solution is better than that obtained by Kundu et al. [19] using different ranking approach for the objective function and the constraints (Subsections 5.1 and 5.2, pp. 2032-2033). Also, to solve the problem we do not require any condition on availability or on total conveyance capacity. Since the proposed method is for fuzzy MOMISTP, it is also applicable for multiobjective transportation problems, solid transportation problems, multiobjective solid transportation problem, multi-item transportation problems, and multiobjective solid transportation problems in fuzzy environment.

\section{Conflict of Interests}

The authors declare that there is no conflict of interests regarding the publication of this paper.

\section{Acknowledgments}

The authors are thankful to the reviewers for their valuable comments and suggestions, which improved the presentation of the paper. The first author is also thankful to CSIR, Government of India, for providing financial support.

\section{References}

[1] F. L. Hitchcock, "The distribution of a product from several sources to numerous localities," Journal of Mathematics and Physics, vol. 20, pp. 224-230, 1941.

[2] E. D. Schell, "Distribution of a product by several properties," in Proceedings of the 2nd Symposium in Linear Programing, DCS/Comptroller, HQ US Air Force, pp. 615-642, Washington, DC, USA, 1955.

[3] K. B. Haley, "The solid transportation problem," Operations Research, vol. 10, pp. 448-463, 1962.

[4] H.-J. Zimmermann, "Fuzzy programming and linear programming with several objective functions," Fuzzy Sets and Systems, vol. 1, no. 1, pp. 45-55, 1978.

[5] B. Liu and Y.-K. Liu, "Expected value of fuzzy variable and fuzzy expected value models," IEEE Transactions on Fuzzy Systems, vol. 10, no. 4, pp. 445-450, 2002.

[6] S. Islam and T. K. Roy, "A new fuzzy multi-objective programming: entropy based geometric programming and its application of transportation problems," European Journal of Operational Research, vol. 173, no. 2, pp. 387-404, 2006.

[7] A. Gupta, A. Kumar, and A. Kaur, "Mehar's method to find exact fuzzy optimal solution of unbalanced fully fuzzy multiobjective transportation problems," Optimization Letters, vol. 6, no. 8, pp. 1737-1751, 2012. 
[8] P. Gupta and M. K. Mehlawat, "An algorithm for a fuzzy transportation problem to select a new type of coal for a steel manufacturing unit," TOP, vol. 15, no. 1, pp. 114-137, 2007.

[9] C. R. Bector and S. Chandra, Fuzzy Mathematical Programming and Fuzzy Matrix Games, vol. 169 of Studies in Fuzziness and Soft Computing, Springer, Heidelberg, Germany, 2010.

[10] L. A. Zadeh, "Fuzzy sets," Information and Computation, vol. 8, pp. 338-353, 1965.

[11] Y. Li, K. Ida, and M. Gen, "Improved genetic algorithm for solving multiobjective solid transportation problem with fuzzy numbers," Japanese Journal of Fuzzy Theory and Systems, vol. 9, no. 2, pp. 197-211, 1997.

[12] A. K. Bit, M. P. Biswal, and S. S. Alam, "Fuzzy programming approach to multiobjective solid transportation problem," Fuzzy Sets and Systems, vol. 57, no. 2, pp. 183-194, 1993.

[13] M. Gen, K. Ida, Y. Li, and E. Kubota, "Solving bicriteria solid transportation problem with fuzzy numbers by a genetic algorithm," Computers and Industrial Engineering, vol. 29, no. 1-4, pp. 537-541, 1995.

[14] A. Ojha, B. Das, S. Mondal, and M. Maiti, "An entropy based solid transportation problem for general fuzzy costs and time with fuzzy equality," Mathematical and Computer Modelling, vol. 50, no. 1-2, pp. 166-178, 2009.

[15] Q. Cui and Y. Sheng, "Uncertain programming model for solid transportation problem," Information, pp. 342-348, 2012.

[16] A. Baidya, U. K. Bera, and M. Maiti, "Multi-item interval valued solid transportation problem with safety measure under fuzzystochastic environment," Journal of Transportation Security, vol. 6, no. 2, pp. 151-174, 2013.

[17] A. Baidya, U. K. Bera, and M. Maiti, "Solution of multi-item interval valued solid transportation problem with safety measure using different methods," OPSEARCH, vol. 51, no. 1, pp. 122, 2014.

[18] A. Kumar and A. Kaur, "Optimal way of selecting cities and conveyances for supplying coal in uncertain environment," Sadhana, vol. 39, no. 1, pp. 165-187, 2014.

[19] P. Kundu, S. Kar, and M. Maiti, "Multi-objective multi-item solid transportation problem in fuzzy environment," Applied Mathematical Modelling, vol. 37, no. 4, pp. 2028-2038, 2013.

[20] P. Kundu, S. Kar, and M. Maiti, "Multi-objective solid transportation problems with budget constraint in uncertain environment," International Journal of Systems Science, vol. 45, no. 8, pp. 1668-1682, 2014.

[21] A. Kaufmann and M. M. Gupta, Introduction to Fuzzy Arithmetics: Theory and Applications, Van Nostrand-Reinhold, New York, NY, USA, 1985.

[22] T.-S. Liou and M. J. Wang, "Ranking fuzzy numbers with integral value," Fuzzy Sets and Systems, vol. 50, no. 3, pp. 247-255, 1992.

[23] J. J. Buckley, T. Feuring, and Y. Hayashi, "Solving fuzzy problems in operations research: inventory control," Soft Computing, vol. 7, pp. 121-129, 2002.

[24] S. Kikuchi, "A method to defuzzify the fuzzy number: transportation problem application," Fuzzy Sets and Systems, vol. 116, no. 1, pp. 3-9, 2000. 


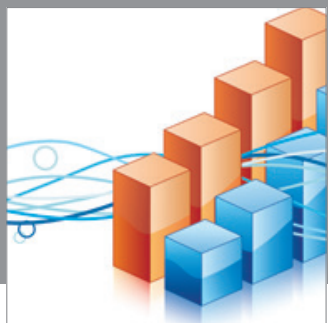

Advances in

Operations Research

mansans

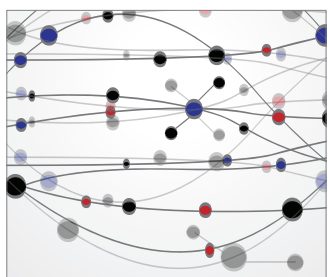

The Scientific World Journal
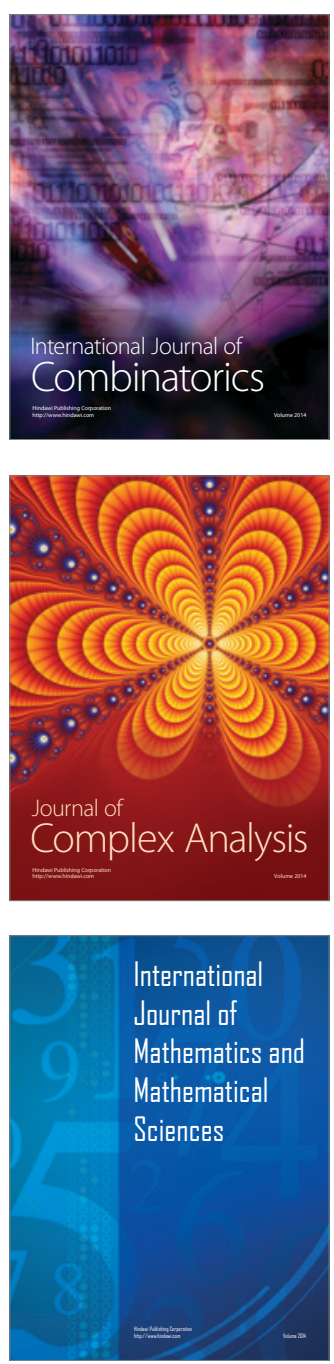
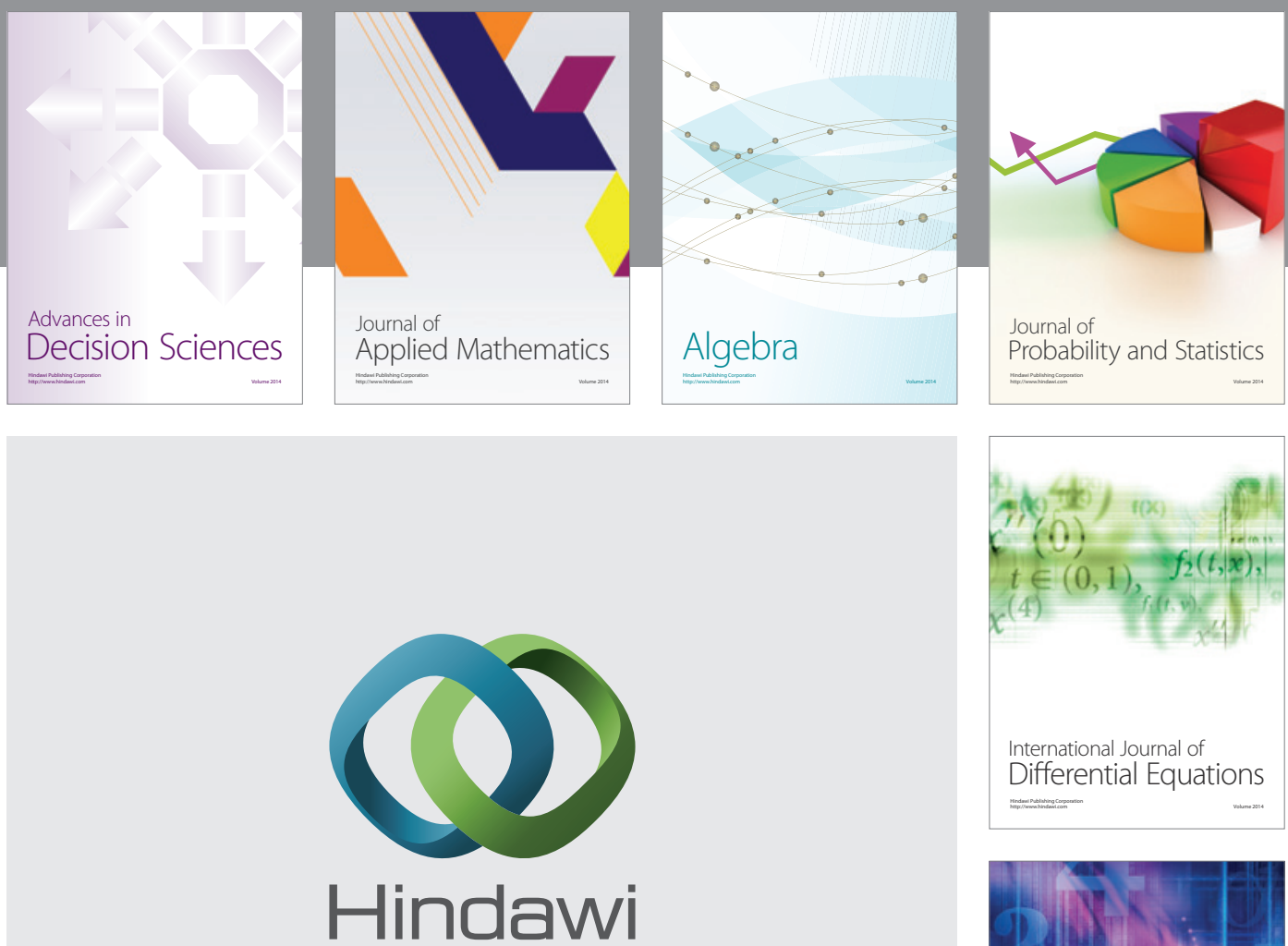

Submit your manuscripts at http://www.hindawi.com
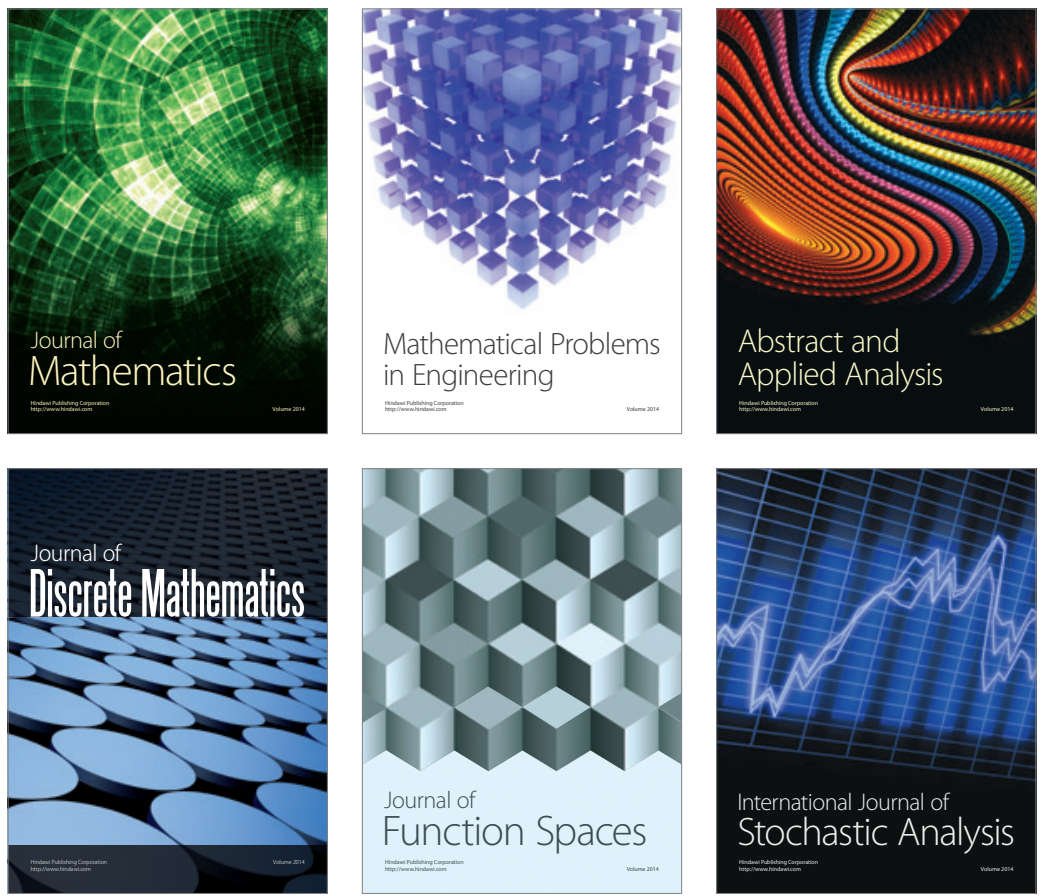

Journal of

Function Spaces

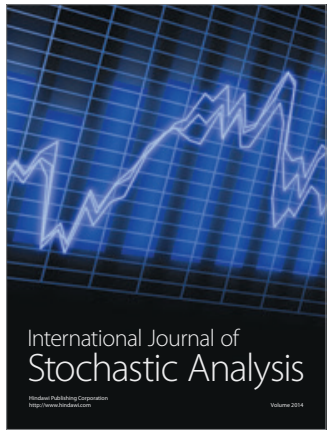

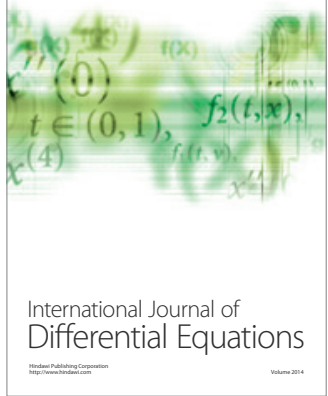
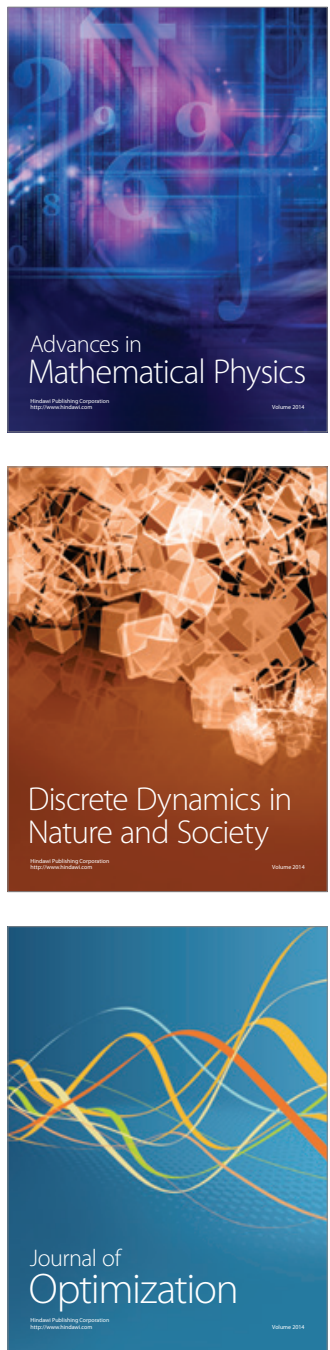